\title{
Recoverable, Reusable, Highly Active, and Sulfur-tolerant Polymer Incarcerated Palladium for Hydrogenation
}

\author{
Kuniaki Okamoto, Ryo Akiyama, and Shū Kobayashi* \\ Graduate School of Pharmaceutical Sciences, The University of Tokyo, Hongo, \\ Bunkyo-ku, Tokyo 113-0033, Japan \\ SUPPORTING INFORMATION
}

$\begin{array}{ll}\text { Table of Contents } & \text { Page }\end{array}$

$\begin{array}{ll}\text { General } & \text { S-1 }\end{array}$

$\begin{array}{ll}\text { Preparation of copolymer (1b) } & \text { S-1 }\end{array}$

$\begin{array}{ll}\text { Preparation of PI Pd (2b) } & \text { S-2 }\end{array}$

Determination of loading of the palladium on PI Pd S-3

$\begin{array}{ll}\text { Typical Procedures and Spectral Data } & \text { S-3 }\end{array}$

$\begin{array}{lr}\text { References } & \text { S-5 }\end{array}$

\section{General}

Tetramethylsilane $(\delta=0)$ was used as an internal standard for ${ }^{1} \mathrm{H}$ NMR and $\mathrm{CDCl}_{3}(\delta=77.0)$ for ${ }^{13} \mathrm{C}$ NMR. Reactions under a controlled pressure of hydrogen were performed with a stainless reactor. The structures of the known compounds were confirmed by comparison with commercially available compounds or data shown in literature.

2-[2-(4-Vinylphenyl)ethoxymethyl]oxirane (3): To sodium hydride (60\% in mineral oil, $1.92 \mathrm{~g}, 48 \mathrm{mmol})$ suspended in tetrahydrofuran $(40 \mathrm{~mL})$ was added a solution of 2-(4-vinylphenyl)ethanol ${ }^{1}(2.37 \mathrm{~g}, 16 \mathrm{mmol})$ in tetrahydrofuran $(9 \mathrm{~mL})$ at $0{ }^{\circ} \mathrm{C}$. After the mixture was stirred for $1 \mathrm{~h}$ at room temperature, 18-crown-6 (422 
$\mathrm{mg}, 1.6 \mathrm{mmol})$ and epichlorohydrin $(4.44 \mathrm{~g}, 48 \mathrm{mmol})$ were added, and the mixture was further stirred for $12 \mathrm{~h}$. After the mixture was cooled to $0{ }^{\circ} \mathrm{C}$ and diluted with diethyl ether, saturated aqueous ammonium chloride was added to quench the reaction, and the aqueous layer was extracted with diethyl ether. The combined organic layers were dried over sodium sulfate, and the solvent was removed under reduced pressure. The residue was purified by silica gel column chromatography (AcOEt/hexane) to afford 2-[2-(4-vinylphenyl)ethoxymethyl]oxirane (3, $2.88 \mathrm{~g}, 88$ \%). ${ }^{1} \mathrm{H} \mathrm{NMR}\left(\mathrm{CDCl}_{3}\right) \delta=2.58(\mathrm{dd}, 1 \mathrm{H}, J=2.7 \mathrm{~Hz}, 5.0 \mathrm{~Hz}), 2.77(\mathrm{dd}, 1 \mathrm{H}, J=4.1$ $\mathrm{Hz}, 5.0 \mathrm{~Hz}), 2.89$ (t, 2H, $J=7.1 \mathrm{~Hz}), 3.10-3.15(\mathrm{~m}, 1 \mathrm{H}), 3.39$ (dd, 1H, J = 5.7, 11.5 $\mathrm{Hz}), 3.64-3.77(\mathrm{~m}, 3 \mathrm{H}), 5.20(\mathrm{dd}, 1 \mathrm{H}, J=0.9 \mathrm{~Hz}, 10.8 \mathrm{~Hz}), 5.70(\mathrm{dd}, 1 \mathrm{H}, J=0.9 \mathrm{~Hz}$, $17.6 \mathrm{~Hz}), 6.69$ (dd, 1H, $J=10.8 \mathrm{~Hz}, 17.6 \mathrm{~Hz}), 7.18$ (d, 2H, $J=8.1 \mathrm{~Hz}), 7.33$ (d, 2H, $J=8.1 \mathrm{~Hz}) ;{ }^{13} \mathrm{C} \mathrm{NMR}\left(\mathrm{CDCl}_{3}\right) \delta=36.0,44.2,50.8,71.5,72.2,113.2,126.2,129.0$, 135.6, 136.6, 138.4; IR (KBr) 3000, 2924, 2867, 1911, 1812, 1701, 1630, 1512 , 1479, 1407, 1337, 1254, 1205, 1107, 991, 909, $839 \mathrm{~cm}^{-1}$; HRMS (EI) Calcd for $\mathrm{C}_{13} \mathrm{H}_{16} \mathrm{O}_{2}\left(\mathrm{M}^{+}\right)$204.1150, found 204.1130.

Copolymer (1b): Styrene $(6.33$ g, 60.8 mmol), 2-[2-(4vinylphenyl)ethoxymethyl]oxirane $(3,1.55 \mathrm{~g}, 7.6 \mathrm{mmol})$, tetraethyleneglycol mono2-phenyl-2-propenyl ether ${ }^{2}$ (4, $\left.2.36 \mathrm{~g}, 7.6 \mathrm{mmol}\right)$, and 2,2'-azobis(isobutyronitrile) (69.9 $\mathrm{mg}, 0.42 \mathrm{mmol})$ were mixed in chloroform $(10 \mathrm{~mL})$. The mixture was stirred for $48 \mathrm{~h}$ under reflux conditions and then cooled to room temperature. The resulting polymer solution was poured slowly into methanol. The precipitated polymer was filtered and washed with methanol several times and dried for $24 \mathrm{~h}$ under reduced pressure to afford the desired copolymer (1b, $6.72 \mathrm{~g}, 66 \%$ yield). The molar ratio of the components was determined by ${ }^{1} \mathrm{H}-\mathrm{NMR}$ analysis (styrene $: \mathbf{3}: \mathbf{4}=88: 7: 5$ ). Mw: 41,741, Mn: 25,922, Mw / Mn = 1.61 (GPC).

Preparation of polymer incarcerated palladium (PI Pd (2b)): Copolymer (1b, $1.00 \mathrm{~g}$ ) was dissolved in tetrahydrofuran $(20 \mathrm{~mL})$ at room temperature, and to this 
solution was added tetrakis(triphenylphosphine)palladium(0) $\left.\left(\mathrm{Pd}\left(\mathrm{PPh}_{3}\right)_{4}\right), 1.0 \mathrm{~g}\right)$ as a core. The mixture was stirred for $24 \mathrm{~h}$ at this temperature. Hexane $(30 \mathrm{~mL})$ was slowly added to the mixture at $0{ }^{\circ} \mathrm{C}$. Coacervates were found to envelope the core dispersed in the medium. The mixture was left to stand at room temperature for $12 \mathrm{~h}$, and the catalyst capsules were then washed with hexane several times and dried at room temperature for $24 \mathrm{~h}$. Next, the catalyst capsules were stirred at $120{ }^{\circ} \mathrm{C}$ for $2 \mathrm{~h}$ to cross-link the polymer chain. The cross-linked catalyst was then washed with tetrahydrofuran several times and dried at room temperature for $24 \mathrm{~h}$ to give polymer incarcerated palladium (PI Pd 2b, $1.04 \mathrm{~g}, 0.707 \mathrm{mmol} / \mathrm{g}$ of palladium metal was loaded).

Determination of loading of the palladium: PI Pd (2b, $50.0 \mathrm{mg})$ was placed in $50 \mathrm{~mL}$-test tube, and sulfuric acid $(1.0 \mathrm{~mL})$ was added. The mixture was heated at $180{ }^{\circ} \mathrm{C}$ for $30 \mathrm{~min}$, and nitric acid $(0.5 \mathrm{~mL})$ was added. The mixture was further heated for $1 \mathrm{~h}$ to give a clear solution. The solution was diluted with water, and was measured the palladium metal by fluorescence X-ray (XRF) analysis.

\section{A typical experimental procedure for the hydrogenation.}

\section{5,6,7,8-Tetrahydro-2-methoxynaphthalene and 1,2,3,4-Tetrahydro-2-} methoxynaphthalene (12 and 13): PI Pd (2b, $0.025 \mathrm{mmol}, 5 \mathrm{~mol} \%)$ and 2methoxynaphthalene $(79.1 \mathrm{mg}, 0.50 \mathrm{mmol})$ were combined in ethanol $(7 \mathrm{~mL})$. The mixture was stirred for $18 \mathrm{~h}$ at $50{ }^{\circ} \mathrm{C}$ under $70 \mathrm{~atm}$ of hydrogen pressure. The catalyst was filtered and washed with tetrahydrofuran, and the solvents of the filtrate were removed under reduced pressure. The residue was purified by PTLC to afford a mixture of 5,6,7,8-tetrahydro-2-methoxynaphthalene (12) and 1,2,3,4-tetrahydro-2methoxynaphthalene (13) $(81.1 \mathrm{mg}$, conv. $100 \%, \mathbf{1 2} / \mathbf{1 3}=73 / 27)$. Recovered PI Pd was dried under reduced pressure and reused. 
5,6,7,8-Tetrahydro-2-methoxynaphthalene (12): ${ }^{1} \mathrm{H}$ NMR $\left(\mathrm{CDCl}_{3}\right) \delta=1.74-1.79$ (m, 4H), 2.69-2.73 (m, 4H), $3.79(\mathrm{~s}, 3 \mathrm{H}), 6.60(\mathrm{~d}, 1 \mathrm{H}, J=2.6 \mathrm{~Hz}), 6.68(\mathrm{dd}, 1 \mathrm{H}, J=$ $2.6 \mathrm{~Hz}, 8.4 \mathrm{~Hz}), 6.97$ (d, 1H, $J=8.4 \mathrm{~Hz})$.

1,2,3,4-Tetrahydro-2-methoxynaphthalene (13): ${ }^{4} \mathrm{H}$ NMR $\left(\mathrm{CDCl}_{3}\right) \delta=1.74-1.86$ (m, 1H), 2.05-2.09 (m, 1H), 2.68-3.10 (m, 4H), $3.42(\mathrm{~s}, 3 \mathrm{H}), 3.62-3.68(\mathrm{~m}, 1 \mathrm{H})$, , 7.05$7.11(\mathrm{~m}, 4 \mathrm{H})$.

Pioglitazone (8): ${ }^{5} \mathrm{H}$ NMR $\left(D_{M S O}-\mathrm{d}_{6}\right) \delta=1.18(\mathrm{t}, 3 \mathrm{H}, J=7.5 \mathrm{~Hz}), 2.58(\mathrm{q}, 2 \mathrm{H}, J$ $=7.5 \mathrm{~Hz}), 3.04(\mathrm{dd}, 1 \mathrm{H}, J=9.0 \mathrm{~Hz}, 14.1 \mathrm{~Hz}), 3.13(\mathrm{t}, 2 \mathrm{H}, J=6.6 \mathrm{~Hz}), 3.30(\mathrm{dd}, 1 \mathrm{H}$, $J=4.4 \mathrm{~Hz}, 14.1 \mathrm{~Hz}), 4.30(\mathrm{t}, 2 \mathrm{H}, J=6.6 \mathrm{~Hz}), 4.85(\mathrm{dd}, 1 \mathrm{H}, J=4.4 \mathrm{~Hz}, 9.0 \mathrm{~Hz}), 6.86$ $(\mathrm{d}, 2 \mathrm{H}, J=8.6 \mathrm{~Hz}), 7.13(\mathrm{~d}, 2 \mathrm{H}, J=8.6 \mathrm{~Hz}), 7.27(\mathrm{~d}, 1 \mathrm{H}, J=8.1 \mathrm{~Hz}), 7.56(\mathrm{dd}, 1 \mathrm{H}$, $J=2.2 \mathrm{~Hz}, 8.1 \mathrm{~Hz}), 8.36(\mathrm{~s}, 1 \mathrm{H})$.

9,10-Dihydrophenanthrene (9): ${ }^{6}{ }^{1} \mathrm{H}$ NMR $\left(\mathrm{CDCl}_{3}\right) \delta=2.86(\mathrm{~s}, 4 \mathrm{H}), 7.20-7.31(\mathrm{~m}$, $6 \mathrm{H}), 7.72-7.74(\mathrm{~m}, 2 \mathrm{H}) \cdot ;{ }^{13} \mathrm{C} \mathrm{NMR}\left(\mathrm{CDCl}_{3}\right) \delta=29.0,123.7126 .9127 .3128 .1134 .5$ 137.3.

1,2,3,4,5,6,7,8-octahydrophenanthrene (10): ${ }^{6} \mathrm{H} \mathrm{NMR}_{\left(\mathrm{CDCl}_{3}\right)} \delta=1.72-1.79(\mathrm{~m}$, $8 \mathrm{H}), 2.53(\mathrm{~m}, 4 \mathrm{H}), 2.72(\mathrm{~m}, 4 \mathrm{H}), 6.85(\mathrm{~s}, 2 \mathrm{H}) ;{ }^{13} \mathrm{C} \mathrm{NMR}\left(\mathrm{CDCl}_{3}\right) \delta=22.8,23.5,26.2$, $30.0,126.4,134.3,135.2$.

1,2,3,4,4a,9,10,10a-octahydrophenanthrene (11): ${ }^{1} \mathrm{H}$ NMR $\left(\mathrm{CDCl}_{3}\right) \delta=0.90-3.09$ (m, 14H), 7.0-7.30 (m, 4H).

2,4-Diphenyl-2-methylpentane ${ }^{8}:{ }^{1} \mathrm{H}$ NMR $\left(\mathrm{CDCl}_{3}\right) \delta=1.02(\mathrm{~d}, 3 \mathrm{H}, J=6.8 \mathrm{~Hz}), 1.14$ (s, 3H), $1.24(\mathrm{~s}, 3 \mathrm{H}), 1.94(\mathrm{dd}, 1 \mathrm{H}, J=5.0 \mathrm{~Hz}, 14.0 \mathrm{~Hz}), 2.05(\mathrm{dd}, 1 \mathrm{H}, J=7.2 \mathrm{~Hz}$, 14.0 Hz), $2.53(\mathrm{~m}, 1 \mathrm{H}), 7.00-7.29(\mathrm{~m}, 10 \mathrm{H}) ;{ }^{13} \mathrm{C} \mathrm{NMR}\left(\mathrm{CDCl}_{3}\right) \delta=25.1,28.2,31.0$, $37.0,38.4,52.7,125.4,125.5,126.0,127.0,127.9,128.2,149.2,149.3$. 
1,2-Dimethoxy-4-propylbenzene': ${ }^{1} \mathrm{H}$ NMR $\left(\mathrm{CDCl}_{3}\right) \delta=0.94(\mathrm{t}, 3 \mathrm{H}, J=7.3 \mathrm{~Hz})$, 1.57-1.67 (m, 2H), $2.53(\mathrm{t}, 2 \mathrm{H}, J=7.3 \mathrm{~Hz}), 3.84(\mathrm{~s}, 3 \mathrm{H}), 3.86(\mathrm{~s}, 3 \mathrm{H}), 6.70-6.79(\mathrm{~m}$,

$3 \mathrm{H}) ;{ }^{13} \mathrm{C} \mathrm{NMR}\left(\mathrm{CDCl}_{3}\right) \delta=13.7,24.6,37.5,55.6,55.7,111.0,111.7,120.1,135.2$, $146.9,148.6$.

3-Phenylpropanol: ${ }^{1} \mathrm{H}$ NMR $\left(\mathrm{CDCl}_{3}\right) \delta=1.82-1.91(\mathrm{~m}, 2 \mathrm{H}), 2.10(\mathrm{~s}, 1 \mathrm{H}), 2.68(\mathrm{t}$, $2 \mathrm{H}, J=7.7 \mathrm{~Hz}), 3.63(\mathrm{t}, 2 \mathrm{H}, J=6.4 \mathrm{~Hz}), 7.15-7.29(\mathrm{~m}, 5 \mathrm{H}) ;{ }^{13} \mathrm{C} \mathrm{NMR}\left(\mathrm{CDCl}_{3}\right) \delta=$ $32.0,34.1,62.2,125.8,128.3,128.4,141.8$.

Phenyl $\boldsymbol{\beta}$-D-glucopyranoside: ${ }^{1} \mathrm{H}$ NMR $\left(\mathrm{DMSO}^{-} \mathrm{d}_{6}\right) \delta=3.10-3.38(\mathrm{~m}, 4 \mathrm{H}), 3.41-$ 3.49 (m, 1H), 3.68 (ddd, 1H, $J=1.8 \mathrm{~Hz}, 5.3 \mathrm{~Hz}, 11.7 \mathrm{~Hz}), 4.54$ (t, 1H, $5.7 \mathrm{~Hz}), 4.84$ $(\mathrm{d}, 1 \mathrm{H}, J=7.3 \mathrm{~Hz}), 5.00(\mathrm{~d}, 1 \mathrm{H}, J=5.1 \mathrm{~Hz}), 5.06(\mathrm{~d}, 1 \mathrm{H}, J=4.4 \mathrm{~Hz}), 5.28(\mathrm{~d}, 1 \mathrm{H}, J$ $=4.8 \mathrm{~Hz}), 6.95-7.03(\mathrm{~m}, 3 \mathrm{H}), 7.25-7.31(\mathrm{~m}, 2 \mathrm{H}) ;{ }^{13} \mathrm{C} \mathrm{NMR}\left(\mathrm{DMSO}_{6}\right) \delta=60.7$, $69.7,73.2,76.6,77.0,100.4,116.2,121.7,129.4,157.5$.

1,2,3,4-Tetrahydroquinoline ${ }^{10}:{ }^{1} \mathrm{H}$ NMR $\left(\mathrm{CDCl}_{3}\right) \delta=1.87-1.95(\mathrm{~m}, 2 \mathrm{H}), 2.76(\mathrm{t}$, $2 \mathrm{H}, J=6.4 \mathrm{~Hz}), 3.24-3.28(\mathrm{~m}, 2 \mathrm{H}), 3.70(\mathrm{brs}, 1 \mathrm{H}), 6.44(\mathrm{~d}, 1 \mathrm{H}, J=7.7 \mathrm{~Hz}), 6.58(\mathrm{t}$, $1 \mathrm{H}, J=7.5 \mathrm{~Hz}), 6.91-6.97(\mathrm{~m}, 2 \mathrm{H}, J=7.7 \mathrm{~Hz}) ;{ }^{13} \mathrm{C} \mathrm{NMR}\left(\mathrm{CDCl}_{3}\right) \delta=22.2,27.0$, $41.9,114.1,116.8,121.3,126.7,129.4,144.8$.

2,3-Dihydrobenzo[b]thiophene ${ }^{11}:{ }^{1} \mathrm{H}$ NMR $\left(\mathrm{CDCl}_{3}\right) \delta=3.24-3.34(\mathrm{~m}, 4 \mathrm{H}), 6.99(\mathrm{t}$, $1 \mathrm{H}, J=7.2 \mathrm{~Hz}), 7.09(\mathrm{t}, 1 \mathrm{H}, J=7.2 \mathrm{~Hz}), 7.15-7.22(\mathrm{~m}, 2 \mathrm{H})$.

\section{References}

(1) Hirao, A.; Takenaka, K.; Yamaguchi, K.; Nakamura, S.; Yamazaki, N. Polym. Comm. 1983, 24, 339.

(2) Akiyama, R.; Kobayashi, S. J. Am. Chem. Soc., 2003, 125, 3412.

(3) Kruse, L. I.; Cha, J. K. Chem. Comm. 1982, 23, 1329.

(4) Deng, L.; Czerwinski, K.; Cook, J. M. Tetrahedron Lett. 1991, 132, 175.

(5) a) Sohda, T.; Momose, Y.; Meguro, K.; Kawamatsu, Y.; Sugiyama, Y.; Ikeda, H. Arzneim-Forsch., 1990, 40, 37; b) Sugiyama, Y.; Taketomi, S.; Shimura, Y.; Sohda, T.; Meguro, K.; Fujita, T. Arzneim-Forsch., 1990, 40, 253; c) Sugiyama, Y.; Shimura, Y.; Ikeda, H. Arzneim-Forsch., 1990, 
$40,436$.

(6) Yalpani, M.; Koster, R. Chem. Ber. 1990, 719.

(7) Gowland, B. D.; Durst, T. Can. J. Chem. 1979, 57, 1462.

(8) Campelo, J. M.; Chakaraborty, R.; Marinas, J. M. Synt. Commun. 1996, 26, 1639.

(9) Ito, K.; Iida, T.; Ichino, K. Tsunezuka, M.; Hattori, M.; Namba, T. Chem. Pharm. Bull. 1982, 30, 3347.

(10) Sanchez-Delgado, R. A.; Gonzalez, E. Polyhedron 1989, 8, 1431.

(11) a) Bianchini, C.; Meli, A.; Moneti, S,; Oberhauser, W.; Vizza, F.; Herrera, V.; Fuentes, A.; Sanchez-Delgado, R. A. J. Am. Chem. Soc. 1999, 121, 7071; b) Rojas, I.; Linares, F. L.; Valencia, Bianchini, N. C. J. Mol. Catal, 1999, 144, 1. 